\title{
Spatial Adaptive Speckle Filtering Driven by Temporal Polarimetric Statistics and its Application to PSI
}

\author{
Victor D. Navarro-Sanchez, Student Member, IEEE and Juan M. Lopez-Sanchez, Senior Member, IEEE
}

\begin{abstract}
Persistent Scatterers Interferometry (PSI) techniques are designed to measure ground deformations using satellite Synthetic Aperture Radar (SAR) data. They rely in the identification of pixels not severely affected by spatial or temporal decorrelation, which in general correspond to pointlike, persistent scatterers (PS) commonly found in urban areas. However, in urban areas we can find not only PS but also distributed scatterers (DS) whose phase information may be exploited for PSI applications. Estimation of DS parameters require speckle filtering to be applied to the complex SAR data, but conventional speckle filtering approaches tend to mask PS information due to spatial averaging. In the context of single-pol PSI, adaptive speckle filtering strategies based on the exploitation of amplitude temporal statistics have been proposed which seek to avoid spatial filtering on non homogeneous areas. Given the growing interest on Polarimetric PSI techniques, i.e. those using polarimetric diversity to increase performance over conventional single-pol PSI, in this work we propose an adaptive spatial filter driven by polarimetric temporal statistics, rather than single-pol amplitudes. The proposed approach is able to filter DS while preserving PS information. In addition, a new methodology for the joint processing of PS and DS in the context of PSI is introduced. The technique has been tested for two different urban datasets: 41 dual-pol TerraSAR-X images of Murcia (Spain) and 31 full-pol Radarsat-2 images of Barcelona (Spain). Results show an important improvement in terms of number of pixels with valid deformation information, hence denser area coverage.
\end{abstract}

Index Terms-SAR interferometry, polarimetry, persistent scatterers, subsidence, speckle.

\section{INTRODUCTION}

$\mathbf{O}$ $\mathrm{NE}$ of the major sources of error inherent to any SAR system is speckle, produced by the mutual interference of coherent electromagnetic waves when reflected by different elements contained in the resolution cell. Speckle behaves as a granular random noise and is generally reduced by using spatial filtering. However, spatial filtering comes at the cost of resolution loss. In addition, the conventional fixed-size sliding window filters may not be suitable for heterogeneous areas, such as urban environments, where preserving the maximum level of detail is desirable.

Persistent Scatterers Interferometry (PSI) techniques are extensively used for subsidence monitoring of urban areas.

This work has been supported by the Spanish Ministerio de Educación y Competitividad (MINECO) and European Union FEDER funds under project TEC2011-28201-C02-02.

Victor D. Navarro-Sanchez and Juan M. Lopez-Sanchez are with the Signals, Systems and Telecommunication Group (SST), EPS, University of Alicante, P.O.Box 99, E-03080 Alicante, Spain (e-mail: victor.navarro@ieee.org, juanma-lopez@ieee.org).
They are based in the analysis of the interferometric phase of pixels that satisfy some stability requirements during the observation period. Most PSI techniques aim to identify stable, point-like scatterers, usually referred as persistent scatterers (PS), which are generally associated with reflections from man-made structures. However, urban areas are complex environments where we can also find distributed scatterers (DS), that is, groups of neighboring pixels sharing similar reflectivity properties that may be considered as part of the same target. Therefore, a methodology which allows the joint processing of PS and DS would be advantageous. We must take into account that, whilst speckle filtering can contribute to a better characterization of DS, too aggressive filtering strategies may lead to the loss of information on point-like PS. Consequently, an adaptive filtering approach is the option of choice for PSI.

So far, a number of speckle filtering approaches have been proposed in the literature [1][2][3][4] that use spatial statistics to adapt the shape and size of the sliding window, so that only homogeneous, connected areas are averaged. The estimation of spatial statistics for a given coordinate generally implies to evaluate a significant number of neighboring samples. Small vicinity windows can lead to biased estimates, while larger windows incur the risk of mixing statistically unhomogeneous areas, so a tradeoff is required. In either case, response from PS will be masked or corrupted by the rest of samples in the set.

In order to avoid resolution loss, adaptive speckle filtering approaches based on the analysis of temporal statistics, such as DespecKS [5], have been introduced in the framework of single-pol PSI. DespecKS algorithm makes use of the twosample Kolmogorov-Smirnov (KS) test to evaluate whether two neighboring pixels have amplitude data drawn from the same probability density function (p.d.f), and thus they can be considered statistically homogeneous pixels (SHP). Amplitude p.d.f. of a pixel is estimated from the amplitude values at all acquisition times, so no spatial averaging is required. Note that other statistical tests can be applied [6]. However, the extension of DespecKS to multi-channel (polarimetric) data can be problematic, since it requires the estimation a multivariate p.d.f., which in general is not straightforward and requires a significantly larger number of samples. In addition, the KS test is suited to real data (such as the amplitude), what implies making some assumptions when dealing with complex data (e.g. amplitude stability indicates phase stability).

In this paper, a polarimetric adaptive filter driven by temporal statistics is proposed. The filter relies on a likelihood ratio 
test for equality of complex Wishart matrices [7] to determine if two temporal sample coherency matrices (as defined in Section II) are drawn from the same distribution. In addition, a methodology for the joint processing of PS and DS is proposed.

The filter have been tested on urban area for dual-pol TerraSAR-X data (Murcia, Spain), as well as for full-pol Radarsat-2 data (Barcelona, Spain). Deformation velocity maps have been obtained, showing an important improvement in terms of density of pixels with valid deformation information, in comparison with not filtered data, thus confirming the suitability of the approach for its use on PSI.

\section{FORMULATION}

\section{A. Polarimetric Homogeneity Test}

In [8], a likelihood ratio test was used to assess polarimetric stationarity of an area over time. Now, we use a similar approach to check if two polarimetric coherency matrices associated with neighboring pixels follow the same distribution, and therefore they can be considered polarimetrically homogeneous pixels (PHP).

Let $\mathbf{k}$ be the $q$ dimensional target vector obtained by projecting scattering matrix $\mathbf{S}$ of a pixel onto the Pauli basis, as defined in [9]. For full-pol data $(q=3)$ it is given by:

$$
\mathbf{k}=\frac{1}{\sqrt{2}}\left[\begin{array}{c}
H H+V V \\
H H-V V \\
2 H V
\end{array}\right]
$$

where $H H$ and $V V$ stand for the horizontal and vertical copolar channels, respectively, and $H V$ is the crosspolar channel. Notice that we assume $H V=V H$ due to reciprocity. In the case of dual-pol data $(q=2)$ with no crosspolar information, the target vector is reduced to:

$$
\mathbf{k}=\frac{1}{\sqrt{2}}\left[\begin{array}{c}
H H+V V \\
H H-V V
\end{array}\right] .
$$

Let us consider a set of $N$ samples of $\mathbf{k}$ obtained for the same pixel coordinates at different dates, i. e. from different SAR images. We can define their $q \times q$ sample temporal coherency matrix $\mathbf{T}$ as:

$$
\mathbf{T}=\frac{1}{N} \sum_{n=1}^{N} \mathbf{k}_{n} \cdot \mathbf{k}_{n}^{\dagger}
$$

where $\dagger$ stands for the hermitian or conjugated transpose. Under the assumption that, along the stack of $N$ images, $\mathbf{k}$ follows a zero mean, circular complex multivariate Gaussian, denoted as $\mathbf{k} \sim \mathcal{N}_{q}^{\mathbb{C}}(0, \boldsymbol{\Sigma})$, the corresponding sample coherency matrix $\mathbf{T}$ follows a complex Wishart distribution with $N$ degrees of freedom $\mathbf{T} \sim \mathcal{W}_{q}^{\mathbb{C}}(N, \boldsymbol{\Sigma})$. Notice that no spatial averaging is required at this point to compute $\mathbf{T}$, since samples are obtained from the temporal dimension.

Two pixels $i$ and $j$ will be considered PHP if their associated sample coherence matrices $\mathbf{T}_{i}$ and $\mathbf{T}_{j}$ follow the same distribution, i.e. they verify the hypothesis $\boldsymbol{\Sigma}_{i}=\boldsymbol{\Sigma}_{j}$. This hypothesis can be verified, as proposed in [7][10], by means of a likelihood ratio test. Likelihood ratio can be obtained from the determinants of the sample coherence matrices as follows:

$$
\Lambda=\frac{\left|\mathbf{T}_{i}\right|^{N_{i}}\left|\mathbf{T}_{j}\right|^{N_{j}}}{\left|\frac{N_{i} \mathbf{T}_{i}+N_{j} \mathbf{T}_{j}}{N_{i}+N_{j}}\right|^{N_{i}+N_{j}}},
$$

where $\Lambda$ is the likelihood ratio, $N_{i}$ and $N_{j}$ indicate the number of target vector samples used to compute $\mathbf{T}_{i}$ and $\mathbf{T}_{j}$, respectively (generally, $N_{i}=N_{j}=N$ ). Two pixels are considered PHP, with an arbitrarily chosen probability of false alarm $P_{f a}$ if:

$$
\Lambda>c_{\beta} \quad P_{f a}\left(c_{\beta}\right)=P\left(\Lambda \leq c_{\beta}\right)=\beta
$$

Expression (5) requires formulation of $P_{f a}\left(c_{\beta}\right)$, which can not be obtained analytically in an easy way. However, an approximated expression for $P_{f a}\left(c_{\beta}\right)$ has been derived in [7][10]. For a two pixels test with $N_{i}=N_{j}=N$, the probability may be expressed as:

$$
\begin{aligned}
& P_{f a}\left(c_{\beta}\right)=1-\gamma_{i n c}\left(q^{2} / 2,-\rho \log \left(c_{\beta}\right)\right) \\
& -\omega_{2}\left[\gamma_{i n c}\left(q^{2} / 2+2,-\rho \log \left(c_{\beta}\right)\right)-\gamma_{i n c}\left(q^{2} / 2,-\rho \log \left(c_{\beta}\right)\right)\right]
\end{aligned}
$$

with

$$
\begin{aligned}
& \rho=1-\frac{2 q^{2}-1}{4 q N} \\
& \omega_{2}=\frac{q^{2}}{4 \rho^{2}}\left(\frac{q^{2}-1}{6}\left(\frac{2}{N^{2}}-\frac{1}{(2 N)^{2}}\right)-(1-\rho)^{2}\right),
\end{aligned}
$$

where $\gamma_{\text {inc }}(a, b)$ represents the incomplete gamma function of $b$ at order $a$.

Similarly to DespecKS algorithm described in [5] for singlepol data, the proposed polarimetric homogeneity test will be performed for each pixel on all the surrounding pixels inside a previously defined range or window. The pixel under analysis and all its PHP neighbors define the pixel's parameter estimation window (PEW). Pixels that pass the test but are not connected to the pixel under analysis either directly or through other PHPs will be discarded to reduce the probability of mixing responses from different radar targets. In this work, a $15 \times 15$ test window centered on the pixel has been considered.

To determine whether a pixel should be treated as part of a DS or as an isolated pixel, we apply a criterion based on the size of the pixel's PEW, $L$ (number of spatial looks). Pixels with $L$ smaller than a certain number of samples $R$ will be considered isolated pixels for processing, hence suitable for PS analysis. On the other hand, pixels with a sufficiently large PEW ( $L \geq R$ ) will be considered suitable for DS parameter estimation. Fig. 1 shows an example of the number of PHPs $(L)$ identified for each pixel over the area of Barcelona, obtained with the set of 31 Radarsat-2 images described in Section III. Brighter areas correspond to polarimetrically homogeneous regions according to the test. The original test has been replaced by an equivalent one given by $\log \Lambda>\log c_{\beta}$, and an arbitrary threshold of $\log \Lambda>-20$ has been set, which ensures a $P_{f a}<1 \%$. An interesting feature of the proposed test is its great sensitiveness to the presence of dominant scatterers, even if they only appear in 


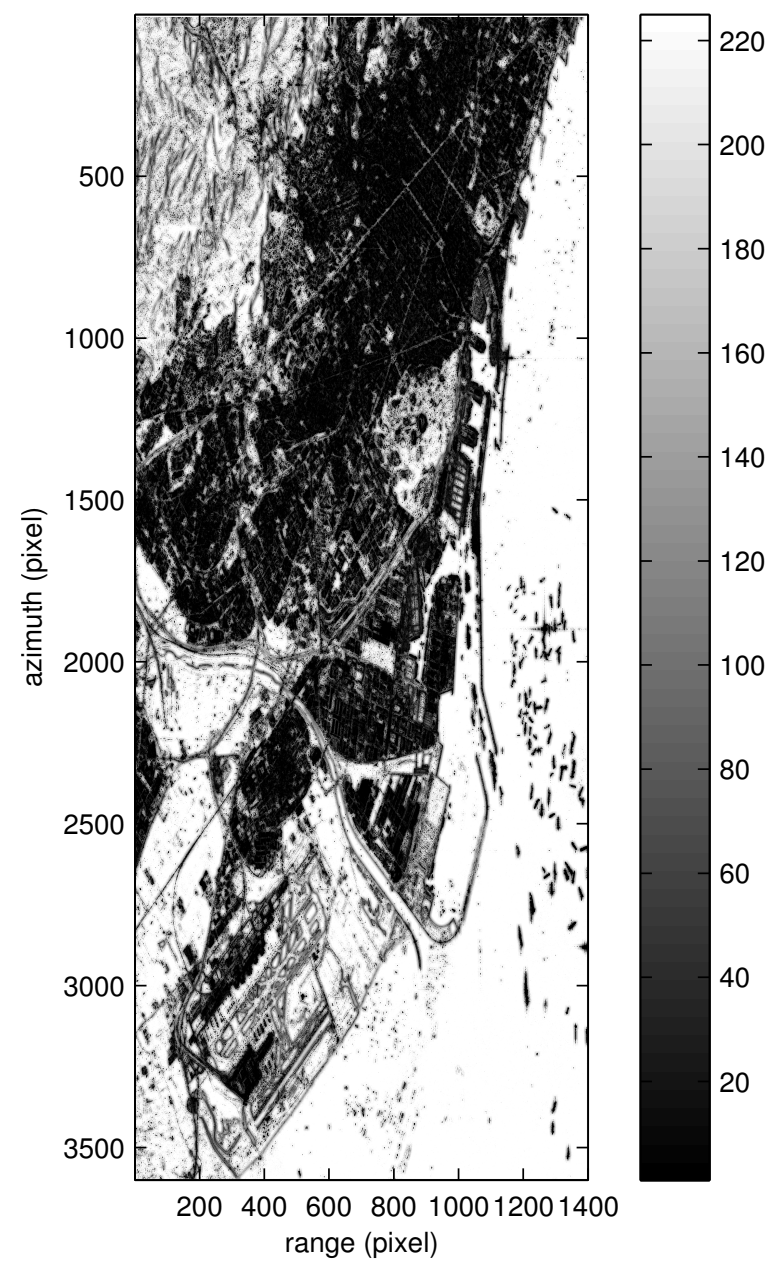

Fig. 1. Number of identified PHP in Barcelona (Spain), for a $\log \Lambda$ threshold of -20 and a test window of $15 \times 15$.

some images (such as, for example, boats in the sea). Hence, pixels dominated by this kind of strong scatterers are generally treated as isolated targets, which is the desired behavior for our application.

The likelihood ratio test (also known as Box's test) is known to perform incorrectly in some cases. To begin with, as aforementioned, this test relies on the assumption that $\mathbf{T}$ samples follow a centered Wishart distribution, so it may fail when this hypothesis does not hold. Particularly, in the case of PS, the assumption that $\mathbf{k}$ samples are zero-mean Gaussian distributed in time is generally not true. More robust versions of the test have been proposed in the literature, which account for departure from normality [11][12]. The test is also known to be biased when the number of samples $(N)$ is small respect to the number of dimensions $(q)$. Alternative asymptotic approximations to the distribution of the likelihood ratio test statistic, for the estimation of $P_{f a}$, can be found in [13]. Nevertheless, from our experience, this simple version of the test provides significantly good results in most cases of study. Experiments carried out in [7] show good performance of the test for full-pol data, with as few as 13 looks for the estimation of sample covariance matrices. Finally, another requirement for the right performance of this test is that $\mathbf{T}$

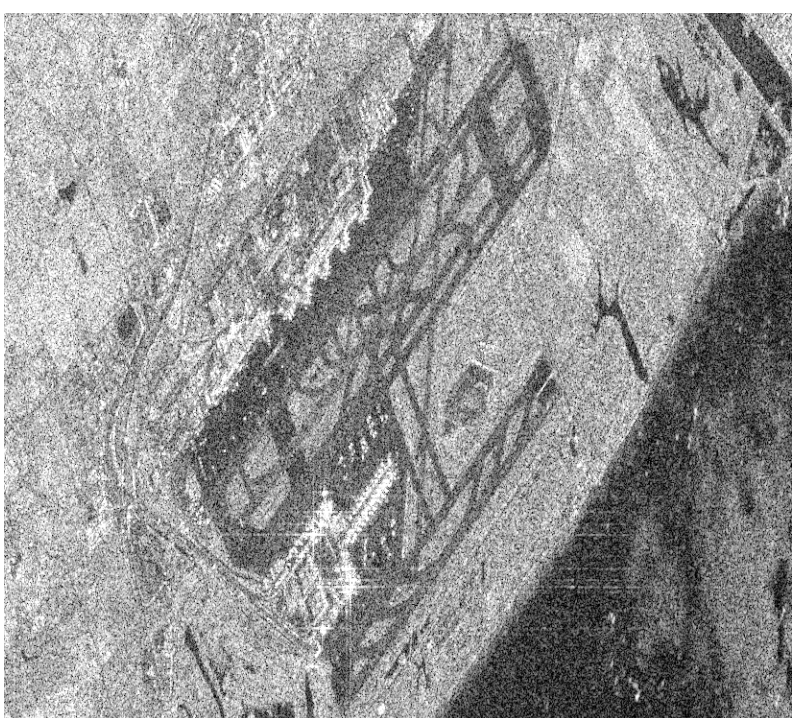

(a)

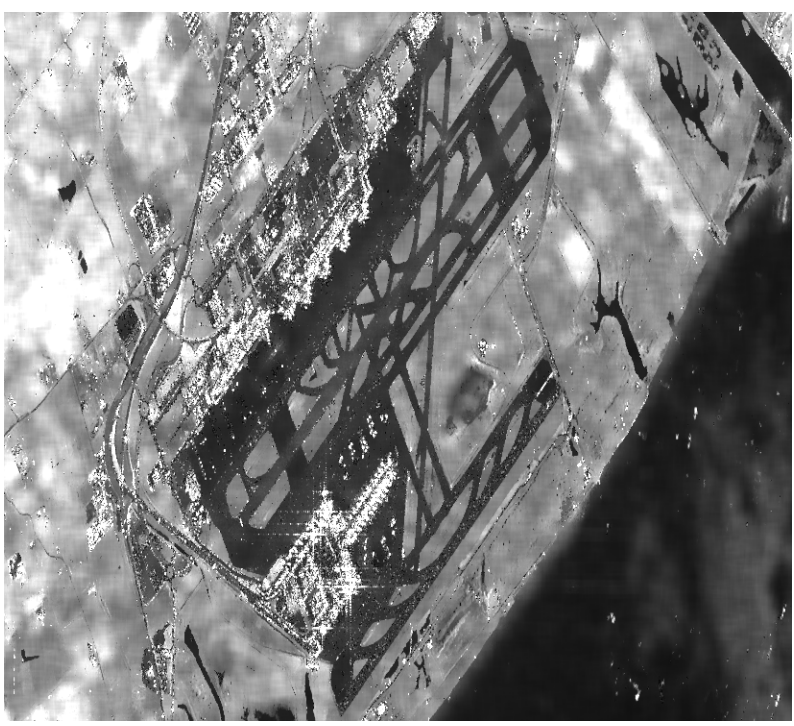

(b)

Fig. 2. Detail of $H H+V V$ amplitude image, original (a) and filtered (b). Filter parameters are: $15 \times 15$ test window size, $\log \Lambda$ threshold of -20 and $R=20$.

matrices have to be full-rank. The rank of the $\mathbf{T}$ matrices implied in the test was evaluated for all the pixels in the available scenes by using a SVD approach, and all of them resulted to be full-rank (rank 2 for dual-pol data and rank 3 for full-pol data), even for pixels selected as PS.

One of the possible applications of this adaptive approach is amplitude despeckling: once the PEWs for each pixel have been determined, we can despeckle amplitude SAR images just by averaging the amplitude values of the PHPs, ignoring those pixels with $L<R$. Fig. 2 shows a detail of a despeckled SAR amplitude image in comparison with the original amplitude image, where a value of $R=20$ has been considered. We can observe how homogeneous areas have been filtered, while high contrast details are preserved. Notice that filtering can also be extended in the temporal dimension, by just applying it to the time-averaged amplitude image. 
Alternatively, for applications where phase information has to be preserved, such as PSI, the adaptive filter can be used to improve the estimation of the polarimetric and interferometric descriptors of DS, while keeping PS unaffected.

In this work we use the proposed filter as a preprocessing step, followed by a polarimetric optimization that will help us to select the best performing or most stable scattering mechanism along time, according to certain quality criterion. Polarimetric optimization step for both PS and DS is summarized in the Section II-B.

Other studies on adaptive multi-temporal speckle filtering strategies can be found in the literature. In [6], different parametric and non-parametric homogeneity tests exploiting single-pol amplitude statistics are evaluated. One of such tests is the Kolmogorov-Smirnov test, also used in the DespecKS filter [5]. In the context of multi-temporal, polarimetric data, an adaptive filter based on binary partition trees have been recently introduced in [14].

\section{B. Polarimetric optimization of PSI}

The main objective of PSI polarimetric optimization is to maximize the quality and number of PS and DS selected as reliable a priori, by optimizing the parameters used as selection criterion. In [15][16][17] we proposed a general framework for PSI polarimetric optimization, starting from the concept of polarimetric (or vector) interferometry introduced in [9]: Let $\mathbf{k}$ be a target vector given by (1). In order to generate an interferogram, $\mathbf{k}$ can be projected onto a unitary complex column vector $\boldsymbol{\omega}$, resulting in $\mu=\boldsymbol{\omega}^{\dagger} \mathbf{k}$, where $\mu$ is a scalar complex scattering coefficient. As a scalar complex, $\mu$ is analogous to single-pol data, so we can make use of any known PSI technique by applying it to $\mu$. Hence, the proposed PSI optimization approach consists in finding, for each pixel, the projection vector $\boldsymbol{\omega}$ that optimizes the parameter considered as quality criterion when computed for $\mu$.

To ease the search of the optimum projection vector (from now on referred as the optimum channel), for fully polarimetric data $\boldsymbol{\omega}$ can be parametrized as follows:

$$
\boldsymbol{\omega}=\left[\begin{array}{l}
\cos (\alpha) \\
\sin (\alpha) \cos (\beta) e^{j \delta} \\
\sin (\alpha) \sin (\beta) e^{j \psi}
\end{array}\right], \quad\left\{\begin{array}{l}
0 \leqslant \alpha \leqslant \pi / 2 \\
0 \leqslant \beta \leqslant \pi / 2 \\
-\pi \leqslant \delta<\pi \\
-\pi \leqslant \psi<\pi
\end{array}\right.
$$

so the problem is reduced to finding four real parameters $\alpha$, $\beta, \delta$ and $\psi$ whose range is finite and known, and whose value is related to the geometric and electromagnetic features of the target [18]. In the following, we will refer to this simple optimization approach as ESPO (Exhaustive Search Polarimetric Optimization). Similarly, in the case of dual-pol data, the two components projection vector can be parameterized as:

$$
\boldsymbol{\omega}=\left[\begin{array}{l}
\cos (\alpha) \\
\sin (\alpha) e^{j \psi}
\end{array}\right], \quad\left\{\begin{array}{l}
0 \leqslant \alpha \leqslant \pi / 2 \\
-\pi \leqslant \psi<\pi
\end{array}\right.
$$

so the search is reduced to two real parameters, $\alpha$ and $\psi$.

Since the reflectivity values for different polarimetric channels can be associated with different scattering mechanisms in the resolution cell located at different heights, hence with different phase centers, we constrain the optimum $\boldsymbol{\omega}$ obtained for each pixel to be the same along all the stack of images. This way we avoid introducing a varying phase term that will result in noisy deformation estimates. This constraint is usually found in the literature as Equal Scattering Mechanisms (ESM) [19], and it can be interpreted as selecting the most stable scattering mechanism over time, according to the chosen criterion of selection.

The most commonly used criterion of selection for PS is the Amplitude Dispersion Index $D_{A}$ [20]. For vector interferometry, $D_{A}$ can be expressed as [15][16]:

$$
D_{A}=\frac{\sigma_{a}}{\bar{a}}=\frac{1}{\overline{\left|\boldsymbol{\omega}^{\dagger} \mathbf{k}\right|} \sqrt{N-1}} \sqrt{\sum_{i=1}^{N}\left(\left|\boldsymbol{\omega}^{\dagger} \mathbf{k}_{i}\right|-\overline{\left|\boldsymbol{\omega}^{\dagger} \mathbf{k}\right|}\right)^{2}}
$$

where $N$ is the total number of images and the overline indicates empirical mean value. Points with $D_{A}$ below a given threshold will be selected as Persistent Scatterer candidates, so in this case our ESPO algorithm will search, for each pixel, the $\boldsymbol{\omega}$ that minimizes its $D_{A}$.

As for DS, in order to evaluate their suitability for PSI it is usual to rely on their average interferometric coherence magnitude $\overline{|\gamma|}$ as an indicator of the quality of the estimated interferometric phases [21][22]. In the context of polarimetric interferometry, $\overline{|\gamma|}$ can be expressed as follows:

$$
\overline{|\gamma|}=\frac{1}{K} \sum_{k=1}^{K}\left|\gamma_{k}\right|, \text { with } \gamma_{k}(\boldsymbol{\omega})=\frac{\boldsymbol{\omega}^{\dagger} \boldsymbol{\Omega}_{i j} \boldsymbol{\omega}}{\sqrt{\boldsymbol{\omega}^{\dagger} \mathbf{T}_{i i} \boldsymbol{\omega}} \sqrt{\boldsymbol{\omega}^{\dagger} \mathbf{T}_{j j} \boldsymbol{\omega}}}
$$

where subscript $k$ denotes the $k$-th interferogram obtained by combining images $i$ and $j$. Polarimetric coherency matrices $\mathbf{T}_{i i}, \mathbf{T}_{j j}$ and polarimetric interferometric cross-correlation matrix $\boldsymbol{\Omega}_{i j}$ are defined as in [9]:

$$
\mathbf{T}_{i i}=E\left[\mathbf{k}_{i} \mathbf{k}_{i}^{\dagger}\right], \quad \mathbf{T}_{j j}=E\left[\mathbf{k}_{j} \mathbf{k}_{j}^{\dagger}\right], \quad \boldsymbol{\Omega}_{i j}=E\left[\mathbf{k}_{i} \mathbf{k}_{j}^{\dagger}\right]
$$

where $E[\cdot]$ is the expectation operator. These expectations cannot be computed in practice, so they will be replaced by their maximum likelihood estimates, given by the empirical mean evaluated using $L$ realizations of the target vectors. In our case the $L$ realizations will be given by the pixel's PEW determined by the proposed adaptive filter, as described in the previous section. This way we guarantee that we are averaging pixels with similar scattering properties, and consequently the usual Gaussian assumption is more likely to hold. In this case, the ESPO algorithm will be applied on each DS to find the the $\boldsymbol{\omega}$ that maximizes $\overline{|\gamma|}$. ESPO and alternative polarimetric optimization approaches are detailed in [17].

Notice that, at this point, we have different criteria of selection for PS and DS. Moreover, it is known that the estimator of $\gamma_{k}$ has a bias depending on the number of looks [23], which in our case can vary for different DS because of the adaptive filter. This is not practical if we want to process jointly PS and DS, since both $D_{A}$ and $\overline{|\gamma|}$ are not directly comparable. In the following subsection we propose an alternative selection criterion that can be used for both PS and DS. 


\section{Joint processing of PS and DS}

In order to process jointly PS and DS, it is convenient to establish a common quality criterion for pixel selection, instead of using $D_{A}$ for PS and $|\bar{\gamma}|$ for DS. The estimated phase standard deviation $\sigma_{\phi}$ has been chosen given its close relationship to both criteria. A pixel (either PS or DS) will be selected as stable if its expected $\sigma_{\phi}$ is lower than a chosen threshold, typically $15^{\circ}(\approx 0.25 \mathrm{rad}$ ).

As demonstrated in [20], $D_{A}$ is an estimator of $\sigma_{\phi}$ for high SNR values (generally, for $D_{A}<0.25$ ), so for PS we can directly consider $D_{A} \approx \sigma_{\phi}$ if we set the selection threshold appropiately. As for DS, $\sigma_{\phi}$ can be estimated from the probability density function of the interferometric phase, $\operatorname{pdf}(\phi)$, which is related to the magnitude of the estimated interferometric coherence $|\gamma|$ and the number of independent looks used in the estimation $L_{e}$, as shown in [24]:

$$
\sigma_{\phi}=\sqrt{\int_{\phi}\left(\phi-\phi_{0}\right)^{2} \operatorname{pdf}(\phi) \mathrm{d} \phi},
$$

with

$$
\begin{aligned}
\operatorname{pdf}(\phi) & =\frac{\Gamma\left(L_{e}+1 / 2\right)\left(1-|\gamma|^{2}\right)^{L_{e}} \gamma \cos \left(\phi-\phi_{0}\right)}{2 \sqrt{\pi} \Gamma\left(L_{e}\right)\left(1-|\gamma|^{2} \cos ^{2}\left(\phi-\phi_{0}\right)\right)^{L_{e}+1 / 2}} \\
& +\frac{\left(1-|\gamma|^{2}\right)}{2 \pi} F_{1}\left(L_{e}, 1 ; 1 / 2 ; \gamma^{2} \cos ^{2}\left(\phi-\phi_{0}\right)\right),
\end{aligned}
$$

where $F_{1}$ is a Gauss hypergeometric function, and the expected phase $\phi_{0}$ can be set to 0 without loss of generality. Note that, when the number of independent samples is large $\left(L_{e}>10\right), \sigma_{\phi}$ can be approximated as [25]:

$$
\sigma_{\phi}=\frac{1}{\sqrt{2 N}} \frac{\sqrt{1-|\gamma|^{2}}}{|\gamma|}
$$

Nevertheless, for a more accurate estimation of $\sigma_{\phi}$ in case of low $L_{e}$ values, we opted for precomputing a look-up table of $\sigma_{\phi}\left(|\gamma|, L_{e}\right)$ based on expression (14), which can be quickly queried at runtime.

For the sake of simplicity, $\sigma_{\phi}$ will be estimated taking into account the average interferometric coherence $|\bar{\gamma}|$, instead of the different values of $\left|\gamma_{k}\right|$ associated with each interferogram $k$.

From expressions (14-15) it is extracted that the lower the coherence magnitude or the number of looks, the higher the expected phase deviation. Notice that, in general, adjacent pixels in a SAR image will be correlated due to averaging, oversampling or other processes, so $L$ spatial samples will correspond, in general, to a smaller number of independent looks, $L_{e}$. In order to account for this, we consider the following approximation:

$$
L_{e} \approx \frac{L}{(\text { ovs_range }) \cdot(\text { ovs_azimuth })},
$$

where ovs_range and ovs_azimuth are the oversampling factors in range and azimuth, respectively. Oversampling factors are obtained as the ratio between theoretical sensor resolution and pixel spacing of the final product. Values of these parameters for the datasets used in this work are shown in Section III.

In [5] an alternative methodology for joint processing of PS and DS is proposed, which relies on a phase triangulation algorithm (PTA) to obtain, for each DS, the $N$ maximum likelihood phase estimates from a set of $K$ interferograms. In that case, the goodness of fit of the PTA is used as an extension, for DS, of the temporal coherence used in several PS-based PSI techniques.

\section{DATASET}

A set of 31 full-pol SLC (Single-Look Complex) images acquired by Radasat- 2 from January 2010 to February 2012 over the urban area of Barcelona (Spain), as well as a set of 41 dual-pol $(H H, V V)$ TerraSAR-X SLC images acquired over Murcia (Spain) from February 2009 to May 2010, have been used to test the proposed algorithms.

Barcelona images have been acquired at a revisit time of 24 days using Radarsat-2 beam mode FQ9: nominal incidence angle of $28^{\circ}$ (near range) to $29.8^{\circ}$ (far range). Nominal azimuth and slant-range resolutions are $7.6 \mathrm{~m}$ and $5.2 \mathrm{~m}$, whereas nominal pixel dimensions are $5.1 \mathrm{~m}$ and $4.7 \mathrm{~m}$, respectively. Therefore, the resulting oversampling factors are 1.49 and 1.11 in azimuth and range. The processing has been applied over a section of the image of $1400 \times 3600$ pixels, where nonurban areas (mainly sea and mountains) have been masked out. A color composite formed by the Pauli average intensities is shown in Fig. 3. The different colors in the composite image provide an insight about the information content of polarimetry. The city is mostly located in the center of the image, showing different dominant channels at different parts. Buildings tend to behave as oriented dihedrals (due to facadeground double reflections), whose polarimetric response depends highly on the orientation angle (OA) [26]. Dihedrals with OA of $45^{\circ}$ have a dominant cross-polar response, whereas $22.5^{\circ}$ rotated dihedrals reflect equally all four polarimetric channels.

As for Murcia images, they have been acquired at a revisit time of 11 days with a mean incidence angle of $37.8^{\circ}$. Azimuth and slant-range resolutions are $6.6 \mathrm{~m}$ and $1.17 \mathrm{~m}$, whereas pixel dimensions are $2.44 \mathrm{~m}$ and $0.91 \mathrm{~m}$. The resulting oversampling factors are 2.7 and 1.28 in azimuth and range. The processing has been applied over a $2000 \times 2000$ portion of the images, centered in the urban area of Murcia. Fig. 4 shows a composite image of the selected scene.

\section{RESULTS}

In this section we evaluate the performance of the proposed approach in terms of density of pixels selected and detail of the generated deformation velocity maps. We compare results obtained by applying conventional PSI to an arbitrary single-pol channel $(H H)$, to the ESPO channel (optimized for minimum $D_{A}$ ), and to ESPO data preprocessed by the proposed adaptive filter. In this experiment we have considered the same filter parameters for both Murcia and Barcelona scenes: $15 \times 15$ PHP search window centered on the pixel, a $\log \Lambda$ threshold of -20 , and $R=20$ minimum number of PHP neighbors for the pixel to be treated as a DS. For comparison purposes, we have also included results obtained by using DespecKS filtering approach [5] on single channel 
TABLE I

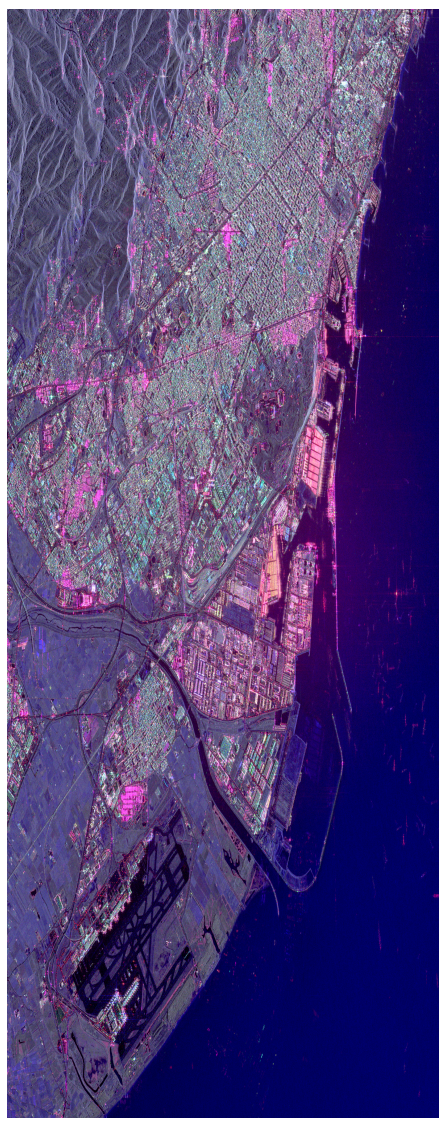

MEASURE POINTS SELECTED FOR DIFFERENT CHANNELS, CONSIDERING A $\sigma_{\phi}$ THRESHOLD OF 0.25

\begin{tabular}{|c|c|c|c|c|}
\hline TerraSAR-X & PS & DS & MP (PS + DS) & $\mathbf{M P / \mathbf { k m } ^ { 2 }}$ \\
\hline HH & $3.60 \%$ & - & $3.60 \%$ & 9968 \\
\hline DespecKS HH & $3.60 \%$ & $0.86 \%$ & $4.45 \%$ & 12321 \\
\hline ESPO (dual-pol) & $8.63 \%$ & - & $8.63 \%$ & 23865 \\
\hline $\log \Lambda$ filter + ESPO & $8.16 \%$ & $5.10 \%$ & $13.26 \%$ & 36682 \\
\hline \hline Radarsat 2 & PS & DS & MP (PS + DS) & MP/km \\
\hline HH & $2.01 \%$ & - & $4.07 \%$ & 425 \\
\hline DespecKS HH & $2.00 \%$ & $13.88 \%$ & $15.88 \%$ & 3364 \\
\hline ESPO (full-pol) & $13.83 \%$ & - & $13.83 \%$ & 2930 \\
\hline $\log \Lambda$ filter + ESPO & $13.36 \%$ & $5.26 \%$ & $18.62 \%$ & 3945 \\
\hline
\end{tabular}

data (HH), with $R=20$ and a Kolmogorov-Smirnov twosample test significance level of $\alpha=0.01$ (1\%).

In order to compare the density of pixels selected by each approach, Table 1 summarizes the output of the selection stage. For the Barcelona scene, sea and mountains have been masked out. A distinction is made between pixels selected and processed as PS and points processed as DS, where applicable. The generic term MP (measure points) is given to the total sum of PS and DS, as in [5]. Selection results are given both in terms of $\%$ of pixels selected from the total pixels of the scene, and in terms of $\mathrm{MP} / \mathrm{km}^{2}$. Note that, due to the different pixel spacing and incidence angle, an area of $1 \mathrm{~km}^{2}$ corresponds to approximately $207 \times 102=21114$ pixels (azimuth $\times$ range) for Radarsat- 2 SLC images, whereas for TerraSAR-X it corresponds to $\approx 410 \times 674=276340$ pixels, and hence the MP/km${ }^{2}$ values differ in one order of

Fig. 3. Composite RGB image of the area under study in Barcelona (Spain) formed by the average intensities. Channels: $\mathrm{R}=H H-V V, \mathrm{G}=2 H V$, $\mathrm{B}=H H+V V$

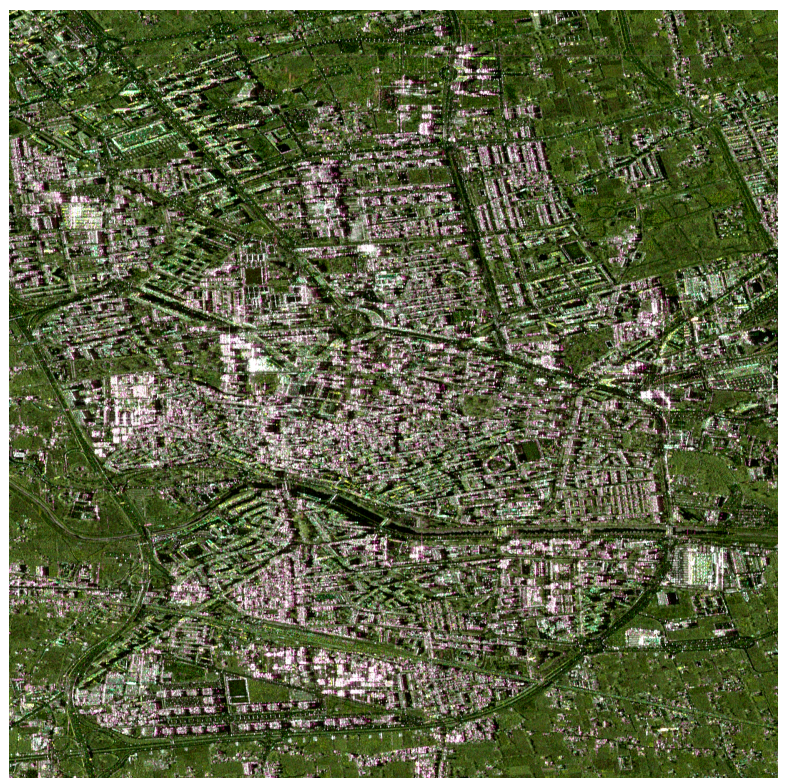

Fig. 4. Composite RGB image of the area under study in Murcia (Spain) formed by the average intensities. Channels: $\mathrm{R}=H H, \mathrm{G}=V V, \mathrm{~B}=H H-V V$ magnitude between the two sensors. As appreciated in the table, the density of total selected pixels, or MP, increases significantly with filtering.

In the case of Murcia TerraSAR-X data, the DespecKS approach applied to only the HH channel does not render as good results as dual-pol ESPO. DespecKS achieves an increase of MP of around $\times 1.24$ with respect to unfiltered $H H$ channel (4.45\% vs. $3.6 \%$ ) due to the selection of new DS. However, given the heterogeneity of the scene and the fine resolution of the sensor, this increase in the number of DS is not so significant as the increase in PS achieved by the ESPO approach, of around $\times 2.4$ with respect to $H H(8.63 \%$ for ESPO compared to $3.60 \%$ for $H H$ ). Now, if we apply the proposed adaptive filter preprocessing to ESPO, the increase is even more spectacular, obtaining 3.7 times more pixels (a total of $13.26 \%$ ) than for $H H$.

For the Barcelona full-pol Radarsat-2 set, the increase of pixels density of ESPO w.r.t. $H H$ is around $\times 6.9(13.83 \%$ for ESPO compared to $2.01 \%$ for $H H$ ). However, in this case the DespecKS filtering approach selects a larger percentage of pixels $(15.88 \%)$, by revealing a significant number of DS. Nevertheless, note that in the ESPO approach all selected pixels are treated as PS, and hence their resolution will not be degraded by filtering. DespecKS, on the other hand, is not contributing to the detection of new PS, but DS. Finally, if we apply the polarimetric filter proposed in this paper to ESPO, we obtain an increase of around $\times 9.27$ in comparison with $H H$ (18.62\% of pixels selected, from which $13.36 \%$ are to be treated as PS). We noticed that in this case, the proposed 


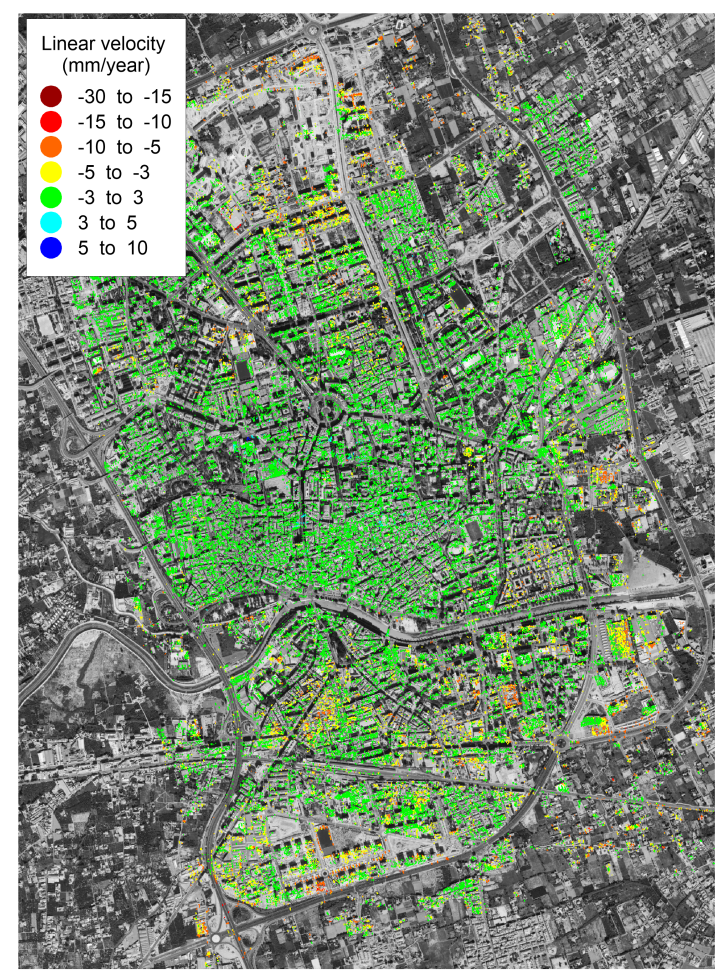

$\mathrm{HH}(3.60 \%)$

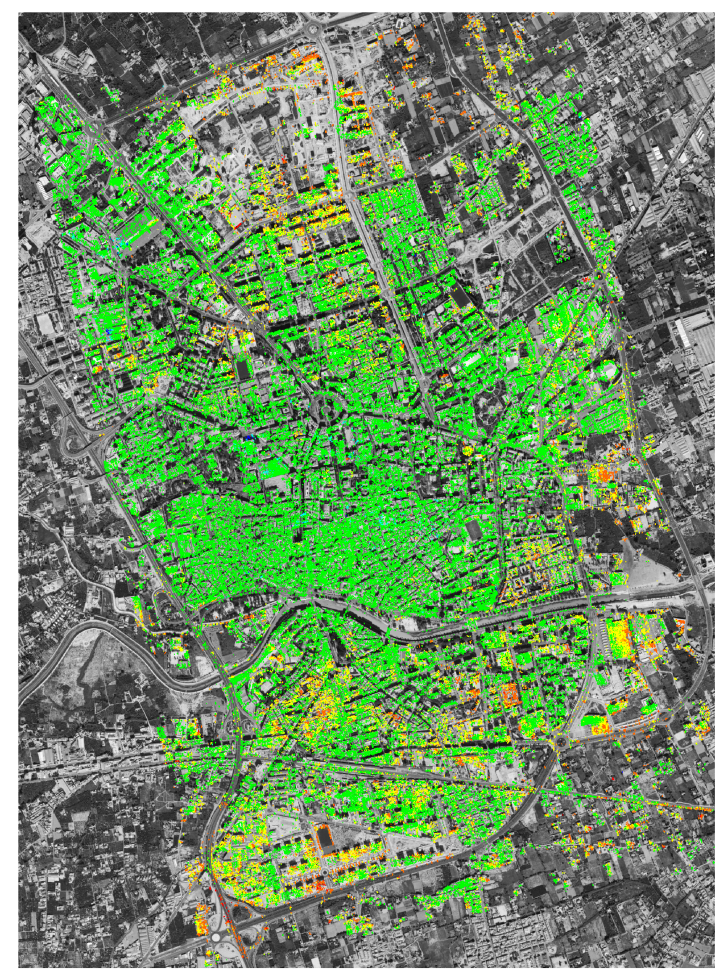

ESPO, Quad-Pol (8.63\%)

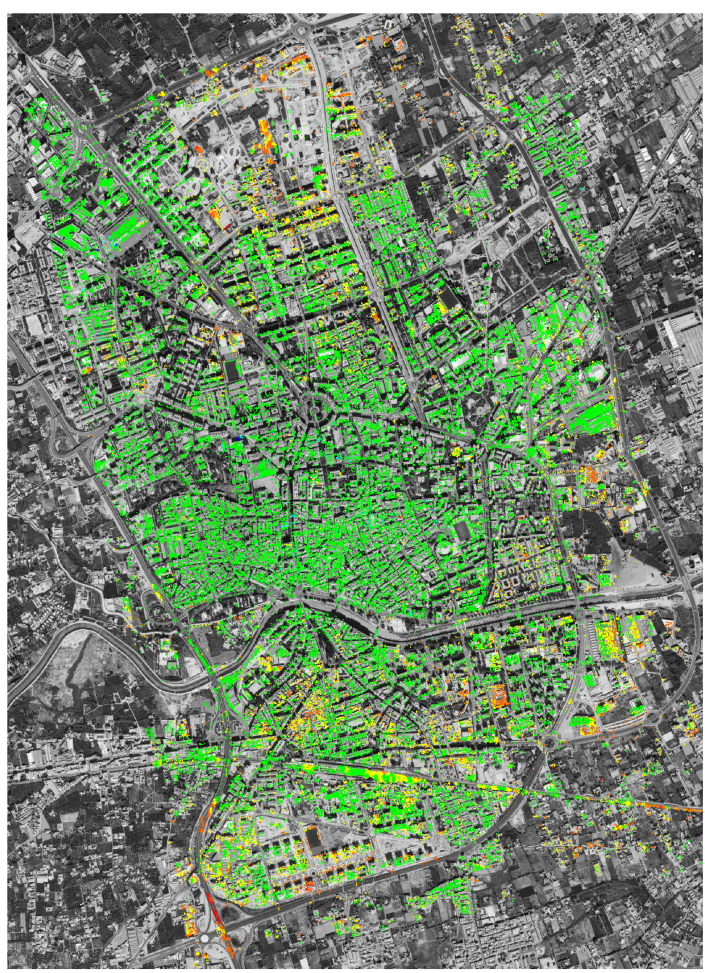

DespecKS, HH (4.45\%)

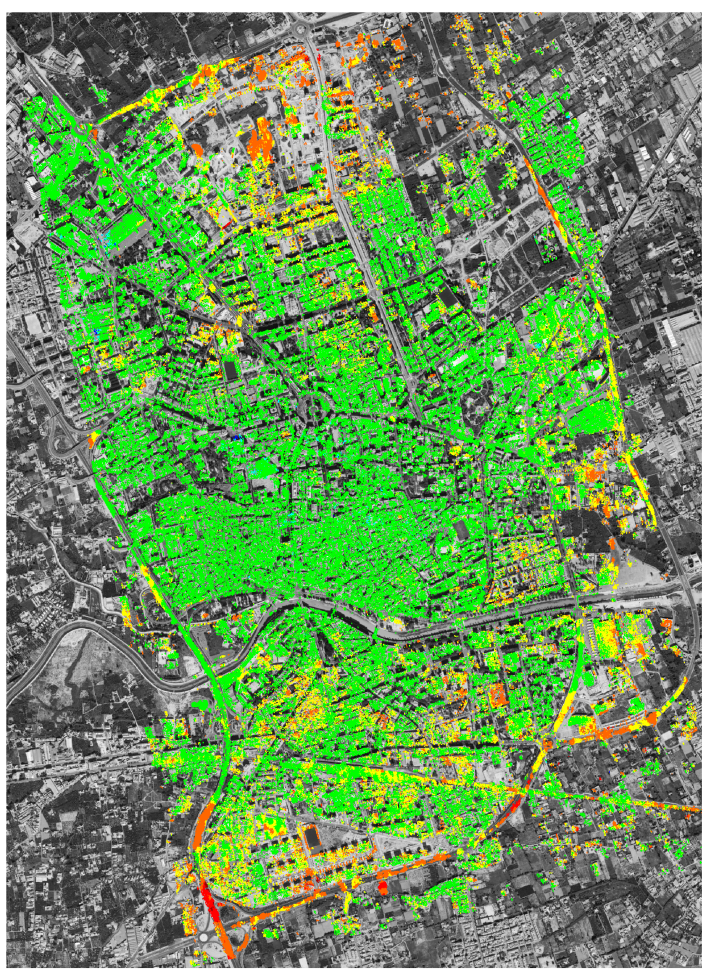

$\log \Lambda$ filter + ESPO Quad-Pol (13.26\%)

Fig. 5. Deformation velocity maps obtained for Murcia scene using different configurations, and percentage of selected pixels. 


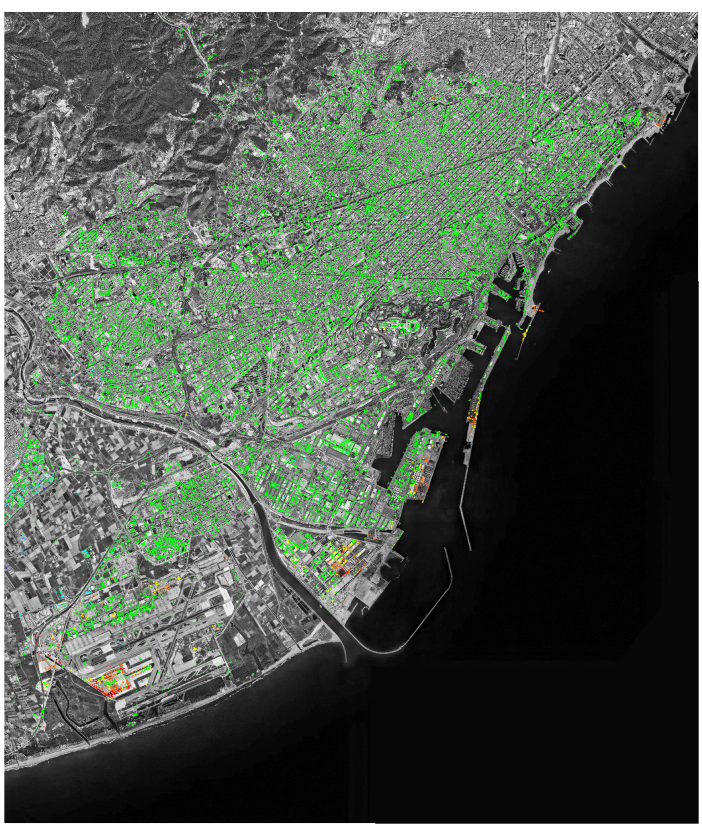

$\mathrm{HH}(2.01 \%)$

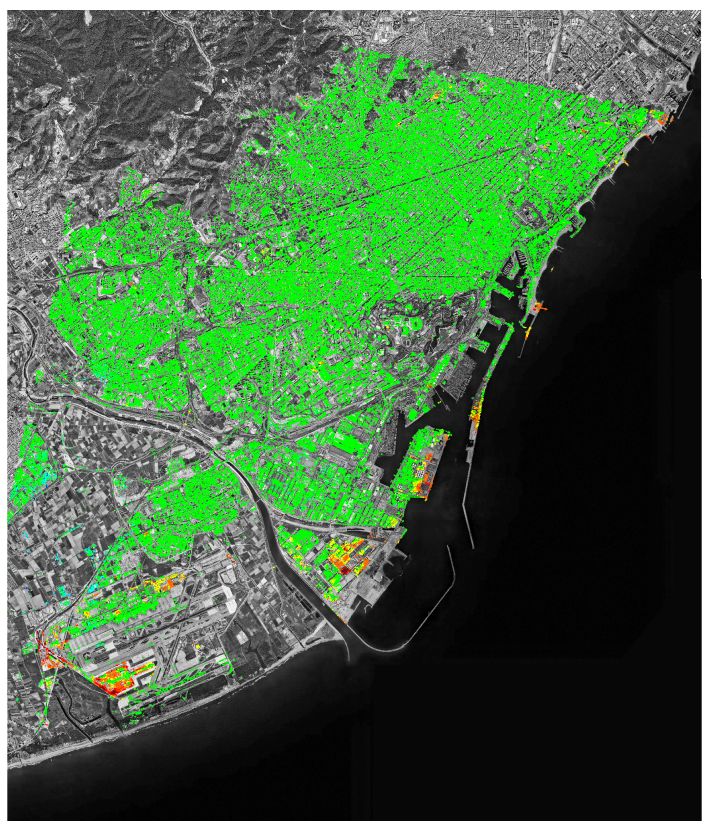

ESPO, Quad-Pol (13.83\%)

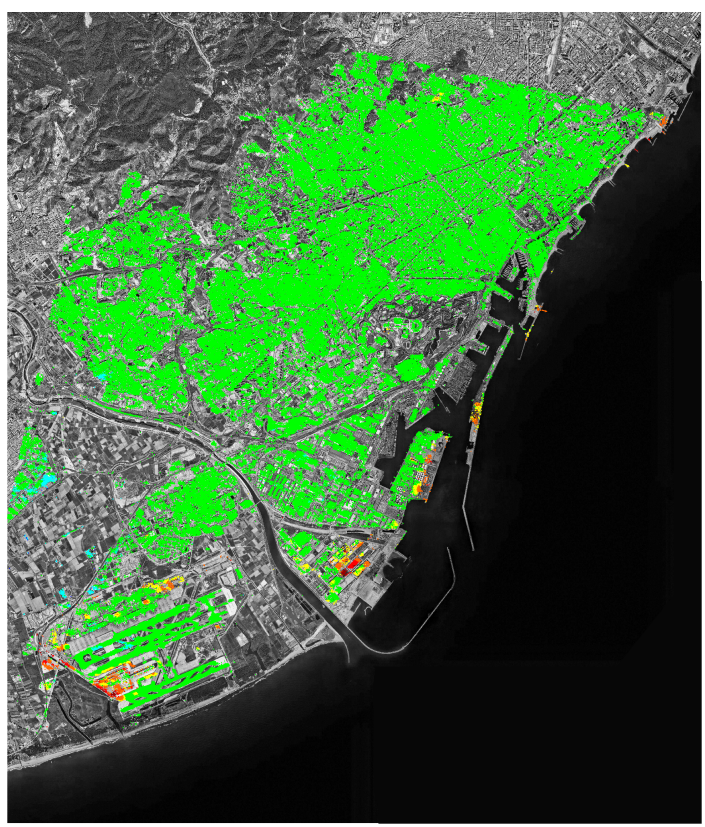

DespecKS, HH (15.88\%)

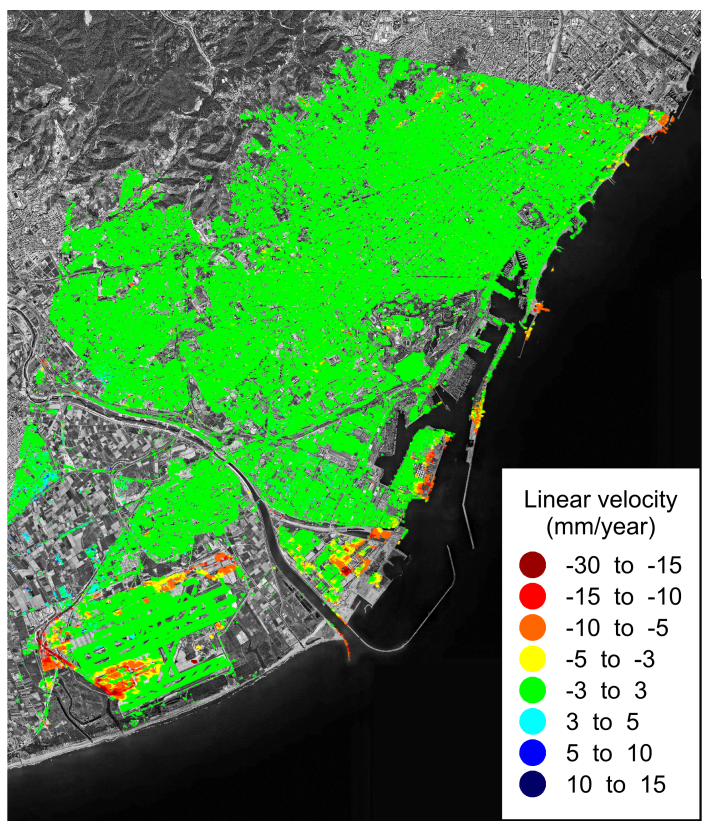

$\log \Lambda$ filter + ESPO Quad-Pol (18.62\%)

Fig. 6. Deformation velocity maps obtained for Barcelona scene using different configurations, and percentage of selected pixels.

filtering approach is more sensitive to changes in the scene than DespecKS, for the parameters considered. Consequently, the average number of homogeneous neighbors per pixel is significantly smaller for the $\log \Lambda$ filter $(\approx 65$ PHP) than for DespecKS approach $(\approx 102$ SHP), and thus a larger number of pixels are treated as PS.

Deformation velocity maps have been generated for both scenes using an implementation of the PSI technique described in [27], which has been adapted to process both PS and DS as described in this paper. Fig. 5 (Murcia) and Fig. 6 (Barcelona) show the obtained velocity maps for each channel, as well as the percentage of points selected.
We can clearly observe in both cases the significant improvement rendered by the inclusion of the proposed adaptive filter. The benefits are specially evident when we consider fully polarimetric data. We can see how man-made, flat homogeneous structures, such as roads or airstrips, are now more likely to be selected in spite of their low backscattering power, since filtering allows us to reduce deviation of phase estimates for DS. Additionally, since PS information is preserved (and enhanced by polarimetric optimization), we still achieve a good characterization of structures such as small buildings. Fig. 7 and Fig. 8 show an augmented view of representative areas containing the aforementioned structures, where we can 


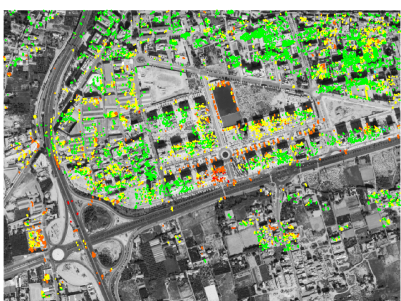

$\mathrm{HH}$

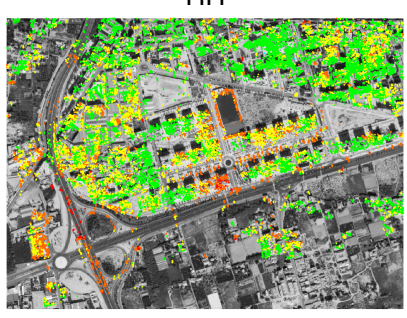

ESPO, Dual-Pol

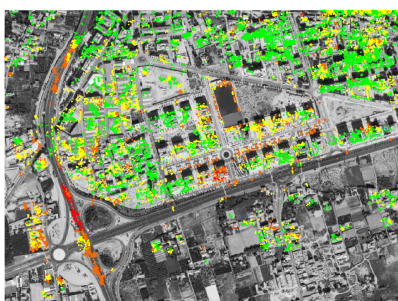

DespecKS, $\mathrm{HH}$

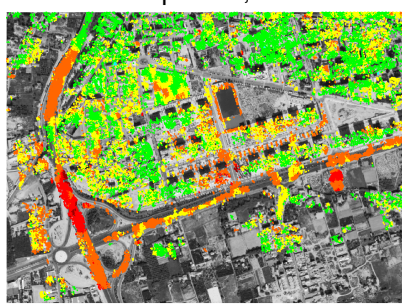

$\log \Lambda$ filter + ESPO Dual-Pol
Fig. 7. Detail of deformation velocity maps, Murcia - Highway bridge. Same color scale as in Fig. 5 is used.

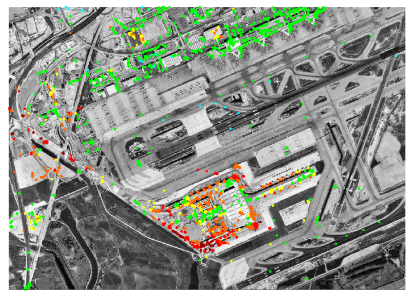

$\mathrm{HH}$

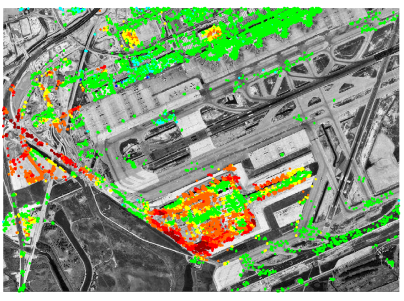

ESPO, Quad-Pol

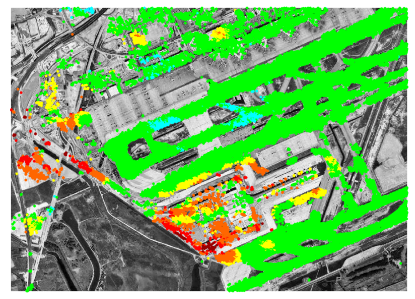

DespecKS, $\mathrm{HH}$

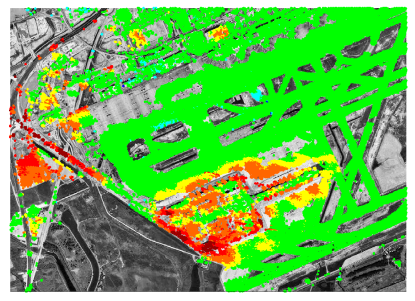

$\log \Lambda$ filter + ESPO Quad-Pol
Fig. 8. Detail of deformation velocity maps, Barcelona - "El Prat" airport. Same color scale as in Fig. 6 is used.

appreciate the new deformation details revealed. Notice that pixel colored markers have been set to a size slightly larger than the resolution cell, in order to ease their visualization.

Validation of obtained results is yet to be addressed, since we do not dispose of the required ground-truth data at the moment, though a qualitative comparison with other works on Murcia area [28] and Barcelona area [29] using different sensors or datasets shows a good agreement with presented results.

Obviously, the inclusion of the filtering step affects the total processing time. Computational cost mainly depends on the type of input data (higher for full-pol than for dual-pol), the number of images and generated interferograms, and the scene features. Generally, the larger the number of pixels treated as DS, the higher the computational cost, since isolated scatterers do not require speckle filtering. $D_{A}$ is calculated in a matter of seconds for the whole scene, whilst coherence estimation on distributed scatterers may take several hours. In addition to the increased computation time due the filter, the additional cost related to the polarimetric optimization step has to be also accounted for. Moreover, due to the larger number of pixels selected for processing, final deformation velocity estimation stages will take longer to compute as well.

We have carried out our experiments on a Dell ${ }^{\mathrm{TM}}$ Workstation equipped with a 12-core AMD Opteron processor $(2.3 \mathrm{GHz})$ and $192 \mathrm{~GB}$ of RAM. Speckle filtering and polarimetric optimization approaches have been written in IDL (Interactive Data Language). The remaining of the PSI processing chain has been implemented in $\mathrm{C}$ language. As expected, the computational cost of the proposed approach is higher for the full-pol Barcelona case of study, because of the higher dimensionality of data and density of DS. Nevertheless, given the larger number of images and interferograms considered for the dual-pol Murcia scene, the difference in total processing time is not so significant between the two cases. Approximate computation times for each stage are summarized in Table 2. For comparison, we also included computation times for our implementation of the DespecKS filtering approach. We can clearly observe that the polarimetric optimization stage is the most time-consuming step of the proposed technique. However, likelihood ratio test is significantly more efficient (around $3 \times$ faster) than the Kolmogorov-Smirnov test used in DespecKS, hence total computing times are in a similar order of magnitude.

These times represent approximately a $25 \%$ of the complete PSI processing time chain. Since computation time is generally not a critical factor for PSI (processing is carried out when a sufficient number of SAR images is available, which may take years depending on the satellite revisit time), the potential benefit in terms of maps pixel density clearly justifies the increase of computational complexity.

\section{Conclusions}

In this work we propose a new spatial polarimetric adaptive speckle filter driven by temporal statistics, which is able to preserve PS information while filtering DS. Additionally, a simple methodology for joint processing of PS and DS is presented, based on a common criterion of quality, the estimated phase standard deviation $\sigma_{\phi}$.

The approach has been tested using dual-pol TerraSAR$\mathrm{X}$ data and full-pol Radarsat-2 data. PSI results show an important improvement in terms of area coverage (i.e. pixels with deformation information) compared with unfiltered data, either single-channel or polarimetrically optimized data. For filtered TerraSAR-X dual-pol data, area coverage is increased by over $\times 1.5$ w.r.t. unfiltered ESPO data, and $\times 3.7$ w.r.t unfiltered single-pol data. As for filtered Radarsat-2 full-pol data, area coverage is increased by $\times 1.35$ w.r.t. unfiltered ESPO data, and $\times 9.3$ w.r.t unfiltered single-pol data. The combination of adaptive filter and polarimetric optimization on full-pol data is the configuration that provides the best results.

Validation of presented results with ground-truth data is yet to be addressed. However, the increase of pixels density will generally contribute to the robustness of PSI approaches, adding redundancy and hence improving the accuracy of the phase estimates. 
TABLE II

APPROXIMATE COMPUTATION TIMES FOR MAIN SPECKLE FILTERING STAGES.

\begin{tabular}{|c|c|c|c|c|}
\hline Murcia (TSX, dual-pol) & Homogeneity test & Polarimetric optimization & Interferogram filtering and pixels selection & Total \\
\hline $\log \Lambda$ filter + ESPO & $5 \mathrm{~h}$ & $13 \mathrm{~h}$ & $4.35 \mathrm{~h}$ & $22.35 \mathrm{~h}$ \\
\hline DespecKS HH & $16 \mathrm{~h}$ & - & $1.5 \mathrm{~h}$ & $17.5 \mathrm{~h}$ \\
\hline \hline Barcelona (RDS2, full-pol) & Homogeneity test & Polarimetric optimization & Interferogram filtering and pixels selection & Total \\
\hline $\log \Lambda$ filter + ESPO & $6 \mathrm{~h}$ & $17.2 \mathrm{~h}$ & $6.7 \mathrm{~h}$ & $29.9 \mathrm{~h}$ \\
\hline DespecKS HH & $17 \mathrm{~h}$ & - & $2.5 \mathrm{~h}$ & $19.5 \mathrm{~h}$ \\
\hline
\end{tabular}

The performance and optimum configuration of the adaptive filter depends mainly on the features of the scene under study, as well as the sensor specifications. As a future research line, performance of the filter on different scenarios as a function of configuration parameters will be carefully revised. Additionally, different tests for PHP discrimination will be studied and compared.

\section{ACKNOWLEDGMENT}

The TerraSAR-X images have been provided by the German Aerospace Center (DLR) in the framework of project GEO0389 of the TerraSAR-X scientific program. RADARSAT-2 Data and Products (c) MacDonald, Dettwiler and Associates Ltd. (2010-12) - All Rights Reserved. RADARSAT is an official trademark of the Canadian Space Agency. We thank Prof. Jordi Mallorquí (Universitat Politècnica de Catalunya) for providing access to the RADARSAT-2 data, which were provided by MDA and CSA in the framework of the scientific project SOAR-EU 6779. Ortophotos have been provided by PNOA - (CInstituto Geográfico Nacional de España.

\section{REFERENCES}

[1] J.-S. Lee, M. R. Grunes, and G. de Grandi, "Polarimetric SAR Speckle Filtering and its Implication for Classification," IEEE Trans. Geosci. Remote Sensing, vol. 37, no. 5, pp. 2363-2373, 1999.

[2] R. Touzi, "A Review of Speckle Filtering in the Context of Estimation Theory," IEEE Trans. Geosci. Remote Sensing, vol. 40, no. 11, pp. 2392 2404,2002

[3] A. Alonso-González, C. López-Martínez, and P. Salembier, "PolSAR Speckle Filtering and Segmentation Based on Binary Partition Tree Representation," in Proc. of POLinSAR'11, Frascati, Italy, Jan. 2011. [Online]. Available: http://www.polinsar2011.com

[4] G. Vasile, E. Trouvé, J.-S. Lee, and V. Buzuloui, "Intensity-Driven Adaptive-Neighborhood Technique for Polarimetric and Interferometric SAR Parameters Estimation," IEEE Trans. Geosci. Remote Sensing, vol. 44, no. 6, pp. 1609-1621, June 2006.

[5] A. Ferretti, A. Fumagali, F. Novali, C. Prati, F. Rocca, and A. Rucci, "A New Algorithm for Processing Interferometric Data-Stacks: SqueeSAR," IEEE Trans. Geosci. Remote Sensing, vol. 49, no. 9, pp. 3460-3470, Sept. 2011.

[6] A. Parizzi and R. Brcic, "Adaptive InSAR Stack Multilooking Exploiting Amplitude Statistics: A Comparison Between Different Techniques and Practical Results," IEEE Geosci. Remote Sensing Lett., vol. 8, no. 3, pp. 441-445, May 2011.

[7] K. Conradsen, A. Nielsen, J. Schou, and H. Skriver, "A Test Statistic in the Complex Wishart Distribution and Its Application to Change Detection in Polarimetric SAR Data," IEEE Trans. Geosci. Remote Sensing, vol. 41, no. 1, pp. 4-19, Jan. 2003.

[8] V. D. Navarro-Sanchez and J. M. Lopez-Sanchez, "Polarimetric Stationarity Criteria Applied to the Selection of Pixel Scatterers Candidates," in Proc. of POLinSAR'11, Frascati, Italy, Jan. 2011. [Online]. Available: http://www.polinsar2011.com

[9] S. R. Cloude and K. P. Papathanassiou, "Polarimetric SAR Interferometry," IEEE Trans. Geosci. Remote Sensing, vol. 36, no. 5, pp. 1551-1565, 1998.
[10] L. Ferro-Famil, A. Reigber, E. Pottier, and W. Boerner, "Scene Characterization Using Subaperture Polarimetric SAR Data," IEEE Trans. Geosci. Remote Sensing, vol. 41, no. 10, pp. 2264-2276, Oct. 2003.

[11] D. E. Tyler, "Robustness and efficiency properties of scatter matrices," Biometrika, vol. 70, no. 2, pp. 411-420, 1983.

[12] T. W. Anderson, An introduction to multivariate statistical analysis. Wiley New York, 2003

[13] C. A. Coelho and F. J. Marques, "Near-exact distributions for the likelihood ratio test statistic to test equality of several variance-covariance matrices in elliptically contoured distributions," Computational Statistics, vol. 27, no. 4, pp. 627-659, 2012.

[14] A. Alonso-González, C. Lopez-Martinez, and P. Salembier, "Temporal polsar image series exploitation with binary partition trees," in Proceedings of IGARSS'12. IEEE, 2012, pp. 1493-1496.

[15] V. D. Navarro-Sanchez, J. M. Lopez-Sanchez, and F. Vicente-Guijalba, "A Contribution of Polarimetry to Satellite Differential SAR Interferometry: Increasing the Number of Pixel Candidates," IEEE Geosci. Remote Sensing Lett., vol. 7, no. 2, pp. 276 -280, Apr. 2010.

[16] V. D. Navarro-Sanchez and J. M. Lopez-Sanchez, "Improvement of Persistent-Scatterer Interferometry Performance by Means of a Polarimetric Optimization," IEEE Geosci. Remote Sensing Lett., vol. 9, no. 4, pp. $609-613$, July 2012.

[17] V. D. Navarro-Sanchez, J. M. Lopez-Sanchez, and L. Ferro-Famil, "Polarimetric approaches for persistent scatterers interferometry," IEEE Trans. Geosci. Remote Sensing, vol. PP, 2013. [Online]. Available: http://dx.doi.org/10.1109/TGRS.2013.2253111

[18] S. R. Cloude, Polarisation: Applications in Remote Sensing, O. U. Press, Ed. Oxford University Press, 2010.

[19] M. Neumann, L. Ferro-Famil, and A. Reigber, "Multibaseline Polarimetric SAR Interferometry Coherence Optimization," IEEE Geosci. Remote Sensing Lett., vol. 1, no. 5, pp. 93-97, Jan. 2008.

[20] A. Ferretti, C. Prati, and F. Rocca, "Permanent scatterers in SAR interferometry," IEEE Trans. Geosci. Remote Sensing, vol. 39, no. 1, pp. 8-20, Jan. 2001.

[21] P. Berardino, G. Fornaro, R. Lanari, and E. Sansosti, "A New Algorithm for Surface Deformation Monitoring Based on Small Baseline Differential SAR Interferograms," IEEE Trans. Geosci. Remote Sensing, vol. 40, no. 11 , pp. 2375-2383, Nov. 2002.

[22] O. Mora, J. J. Mallorquí, and A. Broquetas, "Linear and Nonlinear Terrain Deformation Maps From a Reduced Subset of Interferometric SAR images." IEEE Trans. Geosci. Remote Sensing, vol. 41, no. 10, pp. 2243-2253, Nov. 2003.

[23] R. Touzi, A. Lopes, J. Bruniquel, and W. P. Vachon, "Coherence Estimation for SAR Imagery," IEEE Trans. Geosci. Remote Sensing, vol. 37, no. 1, pp. 135-148, Jan. 1999.

[24] J.-S. Lee, K. W. Hoppel, S. A. Mango, and A. R. Miller, "Intensity and Phase Statitistics of Multilook Polarimetric and Interferometric SAR Imagery," IEEE Trans. Geosci. Remote Sensing, vol. 32, no. 5, pp. 10171028, Sept. 1994.

[25] J. O. Hagberg, L. M. H. Ulander, and J. Askne, "Repeat-Pass SAR Interferometry Over Forested Terrain," IEEE Trans. Geosci. Remote Sensing, vol. 33, no. 2, pp. 331 -340, Mar. 1995.

[26] Z. Shan, H. Zhang, C. Wang, W. An, T. Wu, and X. Chen, "FourComponent Model-Based Decomposition of Polarimetric SAR Data for Special Ground Objects," IEEE Geosci. Remote Sensing Lett., vol. 9, no. 5, pp. 989-993, Sept. 2012.

[27] P. Blanco-Sanchez, J. Mallorqui, S. Duque, and D. Monells, "The Coherent Pixels Technique (CPT): An Advanced DInSAR Technique for Nonlinear Deformation Monitoring," Pure and Applied Geophysics, vol. 165, pp. 1167-1193, 2008.

[28] G. Herrera, R. Tomás, D. Monells, G. Centolanza, J. J. Majorquí, F. Vicente, V. D. Navarro, J. M. Lopez-Sanchez, M. Sanabria, M. Cano, and J. Mulas, "Analysis of subsidence using TerraSAR-X data: Murcia case study," Engineering Geology, vol. 116, pp. 284-295, Sept. 2010. 
[29] O. Monserrat, M. Crossetto, M. Cuevas, and B. Crippa, "The Thermal Expansion Component of Persistent Scatterer Interferometry Observations," IEEE Geosci. Remote Sensing Lett., vol. 8, no. 5, pp. 864-868, Sept. 2011.

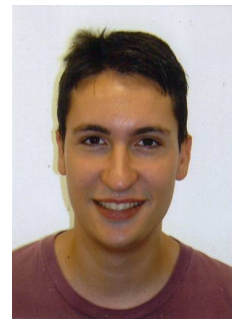

Victor D. Navarro-Sanchez (S'09) was born in Caravaca de la Cruz, Murcia, Spain, in 1982. He received the Ingeniero Técnico degree in Telecommunication Engineering in the University of Alicante, Alicante, Spain in 2004, and the Ingeniero degree in Telecommunication Engineering in the University Miguel Hernández, Elche, Spain in 2009. Since 2009 he works as a predoctoral grantholder with the Signals, Systems and Telecommunication Group of the University of Alicante.

In 2010, he received the Official's Master degree in Computer Technologies in the University of Alicante.

His research interests include polarimetric and interferometric SAR methods for urban and natural environments monitoring and classification.

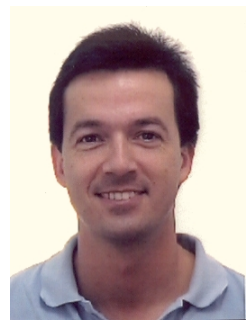

Juan M. Lopez-Sanchez (S'94, M'00, SM'05) was born in Alicante, Spain, in 1972. He received the Ingeniero and Doctor Ingeniero degrees in Telecommunication Engineering from the Technical University of Valencia (UPV), Valencia, Spain, in 1996 and 2000, respectively. From 1998 to 1999 he worked as a predoctoral grantholder at the Space Applications Institute, Joint Research Centre of the European Commission, Ispra, Italy. Since 2000 he leads the Signals, Systems and Telecommunication Group of the University of Alicante, Spain, where he is full professor since November 2011.

His main research interests include microwave remote sensing for inversion of biophysical parameters, polarimetric and interferometric techniques, SAR imaging algorithms and analytical and numerical models for multiple scattering problems.

In 2001, Dr. Lopez-Sanchez received the INDRA award for the best Ph.D. thesis about radar in Spain. From 2006 to 2012 he was the Chair of the Spanish Chapter of the IEEE Geoscience and Remote Sensing Society. He has coauthored more than 40 papers in refereed journals and more than 80 papers and presentations in international conferences and symposia. 